\title{
Molecular epidemiology of dengue viruses in southern China from 1978 to 2006
}

\author{
Weili Wu ${ }^{1 \dagger}$, Zhijun Bai ${ }^{2 \dagger}$, Houqing Zhou ${ }^{3 \dagger}$, Zeng Tu ${ }^{1,4}$, Meiyu Fang ${ }^{2}$, Boheng Tang ${ }^{2}$, Jinhua Liu ${ }^{2}$, Licheng Liu', \\ Jianwei Liu $^{2^{*}}$ and Weijun Chen ${ }^{1,5^{*}}$
}

\begin{abstract}
To investigate molecular epidemiology of dengue viruses (DENV) in southern China, a total of 14 dengue isolates were collected in southern China during each epidemic year between 1978 and 2006 and their full-length genome sequences were obtained by using RT-PCR method. The E gene sequences from additional 6 dengue fever patients in Guangzhou in 2006 were also obtained by using RT-PCR method. Combined with DENVs sequences published in GenBank, phylogenetic analysis and recombination analysis were performed. One hundred and twenty-five E gene sequences and 60 complete genome sequences published in the GenBank were also involved. Phylogenetic analysis showed that there was a wide genetic diversity of DENVs isolated in southern China. DENV-1 strains exist in almost all of the clades of genotype I and IV except the Asia 1 clade of genotype I; DENV-2 stains are grouped into four of the five genotypes except American genotype. DENV-4 strains are grouped into 2 genotypes (I and II). Phylogenetic analysis also showed that all DENV-4 isolates and two DENV-2 isolates were closely related to the prior isolates from neighboring Southeast Asia countries. The DENV-1 strain isolated during the 2006 epidemic is highly homologous to the strains isolated during the 2001 epidemic.

Recombination analysis showed no inter-serotype recombination, but 22 intra-serotype recombination events were found across the 32 complete genomes of all Chinese isolates. The study suggested that dengue fever epidemic in Southern China over the past 30 years presented two important modes, 1) imported-cases-induced endemic prevalence; 2) endogenous epidemic outbreak with natural epidemic focus. Recombination may play an important role in dengue virus evolution and adaptation.
\end{abstract}

\section{Background}

Dengue fever (DF) and two more severe syndromes, dengue shock syndrome (DSS) and dengue hemorrhagic fever (DHF) are important mosquito-borne diseases in tropical and subtropical regions [1,2]. Since the first documented outbreak in 1779 in Jakarta, Indonesia, outbreaks have been documented in tropical and subtropical regions. It has been the maximum public health burden in South-East Asia countries [3]. Dengue epidemiology changes fast among epidemic countries, and keeps on expanding to the non-epidemic area [1]. Since the pathogens were first discovered by Japanese scientists in 1943, dengue viruses (DENV) were isolated from

\footnotetext{
*Correspondence: Ljwgz@21cn.com; Chenwj@genomics.org.cn

+ Contributed equally

${ }^{1}$ Key Laboratory of Genome Sciences and Information, Beijing Institute of Genomics, Chinese Academy of Sciences, Beijing, 100029, China

${ }^{2}$ Guangzhou Medical Research Institute, Yan-ling, Guangzhou, 510507, China Full list of author information is available at the end of the article
}

almost all South-East Asia countries including Thailand, Cambodia, Vietnam, Laos, Myanmar, Malaysia, Philippines, and Indonesia [3]. The dengue virus belongs to Flaviviridae family, and has four closely related but different serotypes (DENV-1 through -4) in nature that are circulating or co-circulating [1,2]. Their genetic diversity remains wide, due to 1 ) absence of a proof-reading capacity in RNA-dependent RNA polymerases [4], 2) emergence of different lineages or clades during epidemic $[5,6], 3)$ increasing natural recombination [7-10], and 4) co-circulation of more than one serotypes in a locality [11-14].

Since imported DF epidemic had been reported in Hankou, Hangzhou, Shanghai and Guangzhou in 1920s and 1940s [15], there was no DF case reported in China till the outbreak occurred in 1978 in Foshan, Guangdong Province, with DENV-4 infection [16]. And then DF was prevalent in Guangdong, Guangxi and Hainan province. A DENV-1 epidemic occurred in 1979 in

\section{() Biomed Central}


Zhongshan, Guangdong Province. This serotype of virus continued causing outbreaks over 2-3 years intervals. It become the dominating serotype and caused the latest outbreak in 2006 [17,18]. DENV-3 epidemic was only recorded once in 1980 at Zhan County, Hainan Island, where in 1985 DENV-2 caused an epidemic during which the first DHF case was reported [19]. After this DENV-2 epidemic, DENV-2 continued to be transmitted into Guangdong, Guangxi and Hainan until 2001, including three outbreaks in Foshan in 1993, 1998 and in Jiangmen in 2001 [20]. The second DENV-4 outbreak occurred in Guangzhou city in 1990 [20]. It was estimated more than 700,540 hospitalized cases with 513 deaths from 1978 to 2007 [15]. Although the first isolate was sampled 30 years ago, dengue epidemic in southern China keeps increasing [15]. Lack of longitudinal research on dengue epidemics has hampered our understanding of dengue molecular origin and evolution in China

In this study, we determined the complete genome sequences of 14 dengue isolates collected in southern China during each epidemic year between 1978 and 2006 and $E$ gene sequences from six patients of Guangdong, 2006. In combination with those published sequences in GenBank, we conducted an extensive molecular epidemiological analysis, aiming to determine where the DENV isolates in China originally came from, and what shaped their evolution.

\section{Materials and methods}

Ethics statement

This research was approved by the Review Board of Guangzhou Medical Research Institute, the Review Board of Shenzhen Second people's Hospital, the Review Board of Beijing Institute of Genomics, the Review Board of Beijing Genomics Institute in Shenzhen and the Review Board of Southwest University. Written informed consent was obtained from the patient for publication of this case report and accompanying images. A copy of the written consent is available for review by the Editor-in-Chief of this journal.

\section{Sera}

Six dengue fever patients' sera were collected in Guangzhou city during the epidemic of DENV-1 in 2006 by Guangzhou Medicine Institute. These sera were collected within 7 days after onset of symptoms and stored at $-20^{\circ} \mathrm{C}$. All sera were tested positive for DEN-1 IgM by indirect immunofluorescence assay at the Guangzhou Medicine Institute.

\section{Viruses}

Fourteen DENV strains were obtained from patients sera in DF epidemic in China during 1978 to 2006
Table 1 Description of DENV isolates sequenced in this study

\begin{tabular}{|c|c|c|c|c|}
\hline Serotype & Isolate $^{a}$ & $\begin{array}{l}\text { Passage history } \\
\text { (host, no. of passages) }^{b}\end{array}$ & Locality & Year \\
\hline & GD03/91 & $\mathrm{SMB}, 3 ; \mathrm{C} 6 / 36,1$ & Guangzhou & 1991 \\
\hline & GD95/95 & SMB, 2; C6/36, 1 & Guangzhou & 1995 \\
\hline & GD01/97 & $\mathrm{SMB}, 1 ; \mathrm{C} 6 / 36,1$ & Chaozhou & 1997 \\
\hline \multirow[t]{6}{*}{ DENV-1 } & GD99/99 & $\mathrm{SMB}, 1 ; \mathrm{C} 6 / 36,1$ & Chaozhou & 1999 \\
\hline & GD54/03 & $\mathrm{SMB}, 1 ; \mathrm{C} 6 / 36,1$ & Guangzhou & 2003 \\
\hline & GD66/03 & $\mathrm{SMB}, 1 ; \mathrm{C} 6 / 36,1$ & Guangzhou & 2003 \\
\hline & GD01/06 & SMB, 1 & Guangzhou & 2006 \\
\hline & GD02/06 & SMB, 1 & Guangzhou & 2006 \\
\hline & GD01/93 & $\mathrm{SMB}, 2 ; \mathrm{C} 6 / 36,1$ & Foshan & 1993 \\
\hline \multirow[t]{3}{*}{ DENV-2 } & GD06/93 & $\mathrm{SMB}, 2 ; \mathrm{C} 6 / 36,1$ & Foshan & 1993 \\
\hline & GD08/98 & SMB, 1; C6/36, 1 & Foshan & 1998 \\
\hline & GD01/01 & $\mathrm{SMB}, 1 ; \mathrm{C} 6 / 36,1$ & Jiangmen & 2001 \\
\hline \multirow[t]{2}{*}{ DENV-4 } & GD07/78 & $\mathrm{SMB}, 2$ & Foshan & 1978 \\
\hline & GD09/90 & SMB, 2; C6/36, 1 & Guangzhou & 1990 \\
\hline
\end{tabular}

${ }^{a}$ All isolates were isolated from patients with primary infection manifesting classic DF symptoms at Guangdong Province, People's Republic of China.

${ }^{b} \mathrm{SMB}$, sucking mouse brain; $\mathrm{C} 6 / 36$, Aedes albopictus cell line.

(Table 1). Sucking BALB/c mice and C6/36 cells were used to isolate viruses from clinical specimens. Serotypes of those isolates were confirmed by indirect immunofluorescence using anti-DENV monoclonal IgG (Guangzhou Medicine Institute, China). These isolates included eight DENV-1 isolates collected from Guangzhou and Chaozhou City of Guangdong province in 1991, 1995, 1997, 1999, 2003 and 2006 epidemic. Four DENV-2 isolates were collected from Fusan and Jiangmen City of Guangdong Province in 1993, 1998 and 2001 epidemic. Two DENV-4 isolates were collected in Foshan City during 1978 epidemic and in Guangzhou city during 1990 epidemic respectively.

\section{Viral RNA extraction and RT-PCR methods}

Viral RNA was extracted using TRIzol reagent (Invitrogen Corp., California, USA) according to the manufacturer's instruction. The cDNA was synthesized by reverse transcription from $10 \mu \mathrm{L}$ of RNA at $42^{\circ} \mathrm{C}$ for 50 min in a $50 \mu \mathrm{L}$ solution containing $50 \mathrm{mM}$ Tris $-\mathrm{HCl}$ (pH 8.3), 75 mM KCl, 3 mM MgCl2, 10 mM DTT, 100 ng of the random hexamer primers, $200 \mathrm{U}$ of Superscript II reverse transcriptase (Invitrogen), $25 \mathrm{U}$ of $\mathrm{RNa}-$ sin (Invitrogen) and $0.5 \mathrm{mM}$ dNTPs. The PCR was performed in a $25 \mu \mathrm{l}$ mixture containing $2 \mu \mathrm{l}$ of cDNA, $10 \mathrm{mM}$ Tris/ $\mathrm{HCl}$ (pH 8.4), $50 \mathrm{mM} \mathrm{KCl,} 2.5 \mathrm{mM} \mathrm{MgCl}$, $100 \mu \mathrm{M}$ each dNTP, 1 U Taq DNA Polymerase (Shanghai Promage Corp., Shanghai, China), $0.25 \mu \mathrm{M}$ each primer (Additional file 1, Table S1-S3). The amplification reactions consisted of an initial denature step of $3 \mathrm{~min}$ at $95^{\circ} \mathrm{C}$, followed by 35 cycles of $30 \mathrm{~s}$ at $94^{\circ} \mathrm{C}, 30 \mathrm{~s}$ at $55^{\circ} \mathrm{C}, 60 \mathrm{~s}$ at $72^{\circ} \mathrm{C}$. 
Viral genome sequencing and $\mathrm{E}$ gene sequencing

The complete genomes of the viruses were sequenced by a PCR-by-PCR strategy. At the same time, Envelope (E) gene from six patients' sera was also amplified. All primers were synthesized by BGI (Beijing Genomics Institute, Beijing, China) according to the reference sequence data of DENV-1 Singapore strain S275/90 [GenBank: M87512], DENV-2 Jamaica strain 1409/88 [GenBank: M20558], and DENV-4 Dominica strain 814669/81 [GenBank: AF326573]. Amplified products were detected by agarose gel electrophoresis and sequenced using ABI 3730xl DNA Analyzers (Applied Biosystems, Foster City, CA).

\section{Assembly of Genome Sequences and Sequence Analysis} Genome assembly was performed independently by distinct operators using Phred-Phrap-Consed [21]. The consensus sequence was yielded exactly with BLASTN and custom script which was written by Perl for all strains. A single contig was obtained for each of the 14 isolates. The single contig was aligned to reference by BLASTN with the E-value of 1e-5. The computation of substitution tables were performed using a custom program written by Perl.

Neighbor-joining trees were constructed using MEGA version 3.1 with the Kimura-2 parameter corrections of multiple substitutions [22]. Reliability of nodes was assessed by bootstrap resampling with 1,000 replicates. One hundred and twenty-five $E$ gene sequences and 60 complete genome sequences published in the GenBank were also involved.

RDP3 was used for recombination analysis. We employed Bootscan, Chimera, GENECONV, MaxChi, and RDP methods incorporated in RDP3 Beta 27 program [23-27]. General recombination settings for all methods were as follows: Sequences were considered as linear, and the highest acceptable P-value was set to 0.01 , and event detected by two or more methods was taken into consideration.

\section{Results}

Nucleotide sequence accession numbers

The determined DENV nucleotide sequences were deposited in GenBank database under the accession numbers FJ196841-FJ196860.

\section{Phylogenetic tree of DENV-1, -2 , and -4 generated by $E$ gene sequences}

To determine the DENV molecular epidemiology in southern China, $145 \mathrm{E}$ gene sequences were used to infer a ML phylogenetic tree (Figure 1), including 1) $20 \mathrm{E}$ gene sequences determined in the study, 2) 47 sequences with China localities available from GenBank, and 3) 78 representative sequences from
GenBank referring to diverse genotype and geographic localities.

The DENV-1 isolates from Guangdong belong to genotype I and IV (Figure 1B). Within genotype I and IV, several sub-genotypes can be identified. Two isolates (CN.GD01/97 and CN.GD99/99) determined in the study clustered together as a single clade with a $96 \%$ bootstrapping support, which was named as a new Asia4 clade. DENV-1 strains isolated in 2001 and 2006 belong to Asia 3 clade in genotype I. However, the stains of 2006 could further be divided into three different sub-clades. Eleven strains of 2006 positioned at the end of the Asia 3 clade and the other four strains positioned at two different sub-clades. Even the eight strains (two isolates and six patients' sera) collected in Guangzhou city during the epidemic of DENV-1 in 2006 also clustered into 2 subclades in Asia 3 clade. All eight strains collected during 2002 and 2003 period at Guangzhou were identical and clustered closely as genotype IV type.

Chinese DENV-2 isolates were characterized into four genotypes except for American genotype (Figure 1C). Broadly, those collected in pre-1990s fell into Asia/ American and Asia II genotypes, while the post-1990s belonged to Asia I and Cosmopolitan genotypes. Two isolates collected in 1998 in Foshan, which belonged to Asia I genotype, were identical to the strain from Thailand in 1993. Three isolates of Jiangmen in 2001 epidemic showed closely identical to the strain from Philippines in 2000 epidemic.

Two DENV-4 epidemics occurred in China in the year of 1978 and 1990. Five isolates collected from the two epidemic years belonged to genotype I and genotype II (Figure 1D). Two isolates collected during 1990 epidemic belonged to genotype I and clustered closely with a strain isolated in 1984 epidemic of Philippines (PH. 12123/84) [GenBank: U18435]. The three other DENV-4 isolates from 1978 epidemic clustered together and fell into genotype II. They resided in phylogenetic tree much closer to the Indonesia isolate 30153 (ID. 30153/ 73) [GenBank: U18428] collected in 1973 when there was a severe dengue epidemic.

\section{Phylogenetic tree of DENV-1, -2 , and -4 generated by ORF sequences}

Based on the 74 complete coding regions (14 determined in the study and 60 from GenBank), we constructed maximum likelihood (ML) phylogenetic tree (Figure 2).

In the study, the whole genome and the single E gene phylogenetic tree are in good coincidence with only little differences. Two DENV-1 isolates, CN.GD01/97 and CN.GD99/99, were assumed to be new members of a defined Asia-4 clade in E gene tree (Figure 1B), while in 


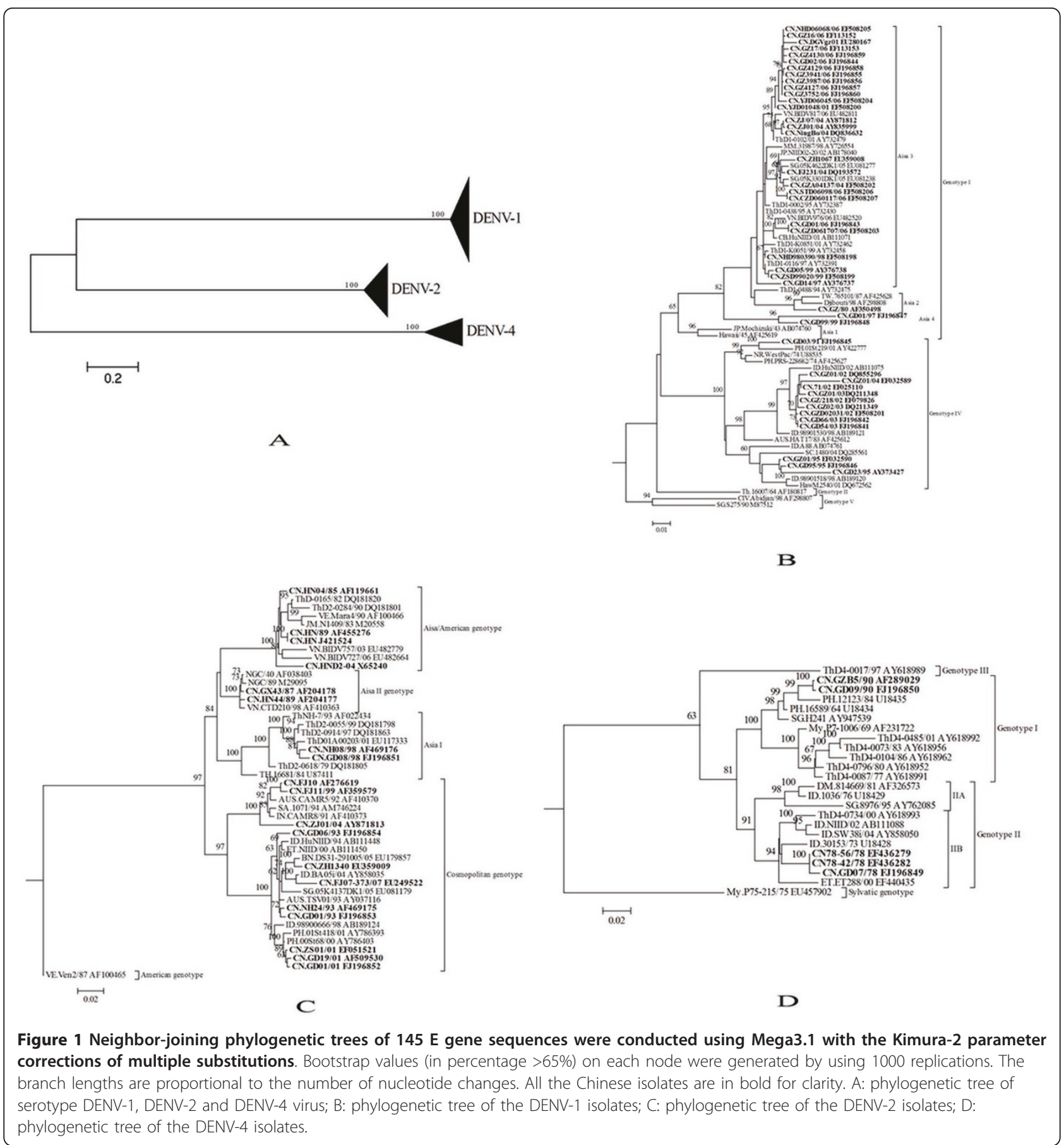

the coding region phylogenetic tree; they clustered into the Asia-3 clade (Figure 2).

\section{Recombination detection}

RDP3 software was employed to examine the potential recombination events of all the Chinese isolates (43 DENV-1,20 DENV-2,2 DENV-3,5 DENV-4) on E gene. Three recombinant events were detected on the $E$ gene of DENV-1 with low P-value $(\mathrm{P}<0.01)$ (Table 2), which was confirmed by phylogenetic construction (Figure 3). Neither DENV-2 nor DENV-4 has any evidence of such recombination across their counterparts. The potential recombination events were also analyzed across the 32 complete ORF of all the Chinese isolates by RDP3 software. No inter-serotype recombination was detected, but 22 intra-serotype potential recombination events (17 for DENV-1, 5 for DENV-2) were confirmed by more than two methods (Table 3). 


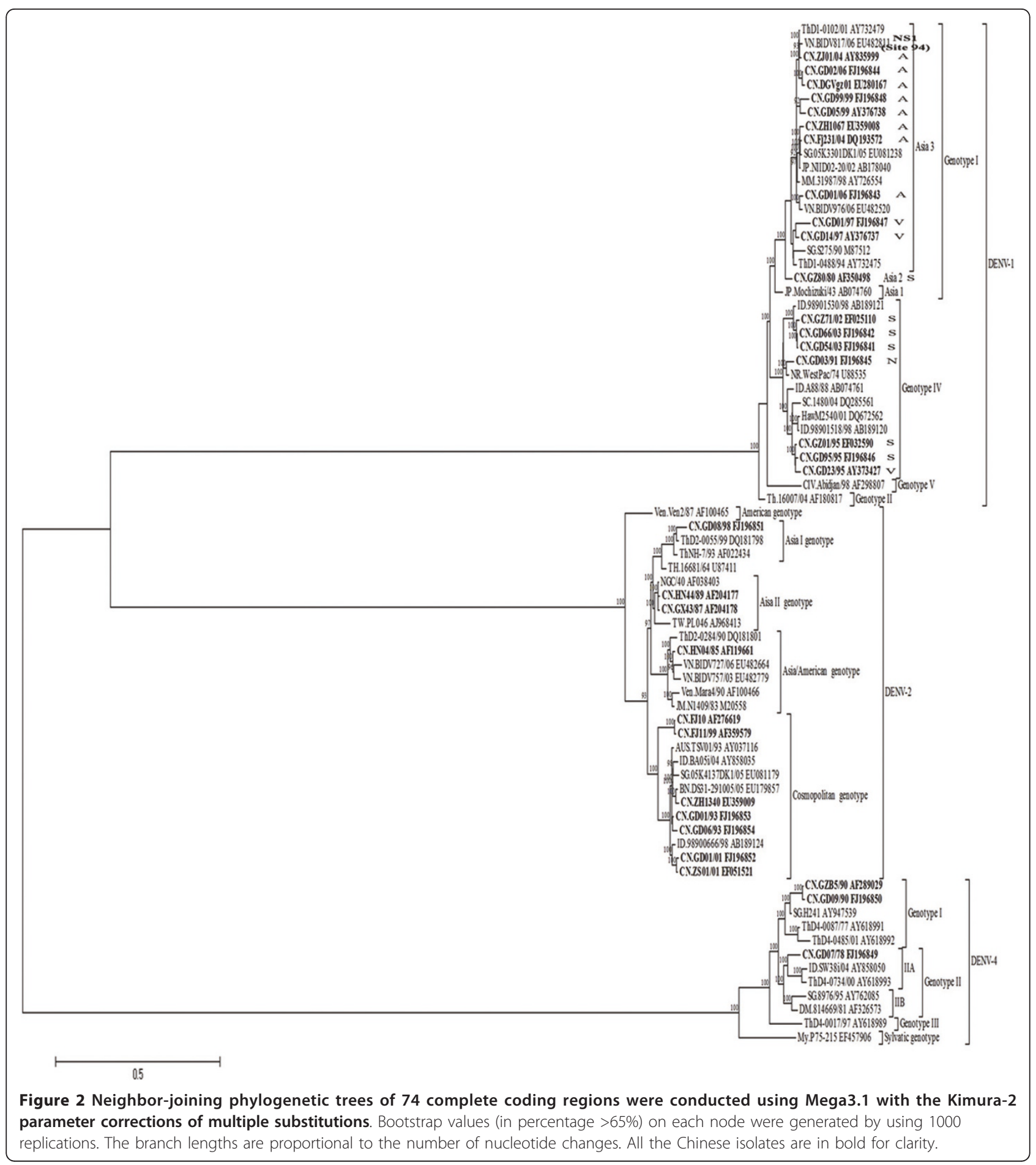

\section{Discussion}

Since the first well documented DF outbreak in Foshan of Guangdong province in 1978, dengue has been reported periodically in southern China, with the latest epidemic at Guangdong province in 2006. In this study, 14. Dengue isolates from 1978 to 2006 were sequenced and analyzed for molecular evolution.
Dengue virus exists as four antigenically distinct viruses designated as serotypes (DENV-1 through DENV-4), belonging to genus Flavivirus of family Flaviviridae. It has a positive-sense RNA genome that is translated as a single polyprotein and posttranslationally cleaved into three structural proteins and seven nonstructural proteins [28]. The envelope protein $(E)$ is 
Table 2 Detected recombination events on the $E$ gene region of Chinese DENV-1*

\begin{tabular}{lllll}
\hline Event & Daughter & Major Parent & Minor Parent & Region $^{\dagger}$ \\
\hline 1 & D1.GD23/95 & D1.GD14/97 & D1.GD95/95 & $1-326$ \\
& (AY373427) & (AY376737) & (FJ196844) & \\
\hline 2 & D1.GD01/97 & D1.NHD98039/98 & D1.GZ02/03 & $60-760$ \\
& (FJ196847) & (EF508198) & (DQ211349) & \\
& D1.GD99/99 & & & $71-760$ \\
& (FJ196848) & & & \\
\hline 3 & D1.GZ01/04 & D1.STD06098 & D1.71/02 & $6-1299$ \\
& (EF032589) & (EF508207) & (EF025110) & \\
\hline
\end{tabular}

* Recombinant region and parental sequences were identified by five procedures (Bootscan, Chimarea, GENECONV, MaxChi, and RDP) integrated in RDP3 Package with a multiple comparison corrected P-value $<0.01$.

${ }^{\dagger}$ Region coordinates are nucleotide positions of detected breakpoints in recombinant E gene sequence (1485bp).

considered to be the immunodominant protein [29]. Dengue viruses also could be divided into different genotypes by the E gene [30,31]. So there is correlation between serotypes and genotypes of Dengue virus. Phylogenetic trees of DENV-1, -2 and -4 generated by ORF and $E$ gene sequences showed that there was a wide genetic diversity of DENVs isolated in southern China (Figure 1, Figure 2). DENV-1 strains exist in almost all of the clades of genotype I and IV but Asia 1 clade of genotype I (Figure 1B, Figure 2). DENV-2 strains are grouped into four genotypes except American genotype (Figure 1C, Figure 2). DENV-4 strains are grouped into 2 genotypes (I and II) (Figure 1D, Figure 2). It also showed that different serotypes and genotypes epidemic prevalence exist in the same city and even during the same epidemic (Figure 1, Figure 2). It indicated that these dengue viruses maybe have different origination.

Some of isolates, especially the first emerging of certain dengue serotype strains in southern China were closely related to those strains which were isolated in prior epidemics from neighboring Southeast Asia countries. It indicated that these dengue epidemics may be imported into China from the neighbor countries. For DENV-2, there were two outbreaks in Foshan in 1993 and 1998. Isolates from 1998 epidemic was closely related to the isolates from 1993 epidemic in Thailand (Figure 1C). The same situation was showed in DENV-4 isolates from 1978 and 1990 epidemics. Isolates from 1978 epidemic was closely related to the isolates from 1973 epidemic in Indonesia (Figure 1D). Isolates form 1990 epidemic was closely related to the isolates from 1984 epidemic in Philippines (Figure 1D). It indicated that these epidemics maybe imported into China from neighbor countries.

Although some data showed the dengue fever were imported from neighbor countries, those epidemics with the same serotype of DENV during continues years showed that endemic infection of dengue circulating locally may be also the important cause of Dengue epidemic in southern China. In the study, two isolates (CN.GD01/97 and CN.GD99/99) clustered together as a new Asia-4 clade (Figure 1B), suggesting that the isolates of 1999 maybe originated from the isolates of 1997. Sequence analysis showed that the DENV-1 strains isolated during the 2003 epidemic were closely related to the strains isolated during the 2002 epidemic of Guangzhou, suggesting that the 2002 strains had evolved in local and eventually cause the epidemic of 2003 (Figure 1B). Similar case was also showed in Guangzhou outbreak of DF in 2006. The DENV-1 strain isolated during the 2006 epidemic is closely related to the strains isolated during the 2001 epidemic (Figure $1 B)$. It suggested that DENV probably circulated in local and caused the epidemics of 2001 and 2006. Moreover, there were several clear divisions within the Chinese 2006 isolates in the tip of the tree, even the eight strains collected in Guangzhou city by us during the epidemic of DENV-1 in 2006 also clustered into 2 subclades in Asia 3 clade, showing viral evolution or increase of genetic complexity even during a single epidemic (Figure 1B).

Recombination plays a role in dengue virus evolution and adaptation [7]. We analyzed the potential recombination events of all the Chinese isolates (43 DENV-1,20 DENV-2,2 DENV-3,5 DENV-4) on E gene. Three recombinant events were detected on the $E$ gene of DENV-1 with low $\mathrm{P}$-value $(\mathrm{P}<0.01)$ (Table 2$)$. The incongruence of phylogenetic trees constructed separately by recombinant regions and non-recombinant regions confirmed the recombinant events (Figure 3). The recombination event 1 showed recombination between present GD14/97 isolate [GenBank: AY376737] as the major parent and the GD95/95 isolate [GenBank: FJ196846] as the minor parent, which led to a recombinant isolate of GD23/95 [GenBank: AY373427](Figure $3 \mathrm{~A})$. The recombination event 2 showed recombination between present NHD98039/98 isolate [GenBank: EF508198] as the major parent and the GZ02/03 isolate [GenBank: DQ211349] as the minor parent, which led to two recombinant isolates of GD01/97 [GenBank: FJ196847] and GD99/99 [GenBank: FJ196848](Figure $3 \mathrm{~B})$. The recombination event 3 showed recombination between present STD06098/06 isolate [GenBank: EF508206] as the major parent and the $71 / 02$ isolate [GenBank: EF025110] as the minor parent, which led to a recombinant isolate of GZ01/04 [GenBank: EF032589] (Figure $3 \mathrm{C}$ ). These studies of recombination provided an important context to understand the evolution of these viruses and sequence diversity generated in viruses in China.

We also analyzed the potential recombination across the 32 complete ORF of all the Chinese isolates, and found no inter-serotype recombination occurred. The results are coincided with previous data $[8,10,32]$. 


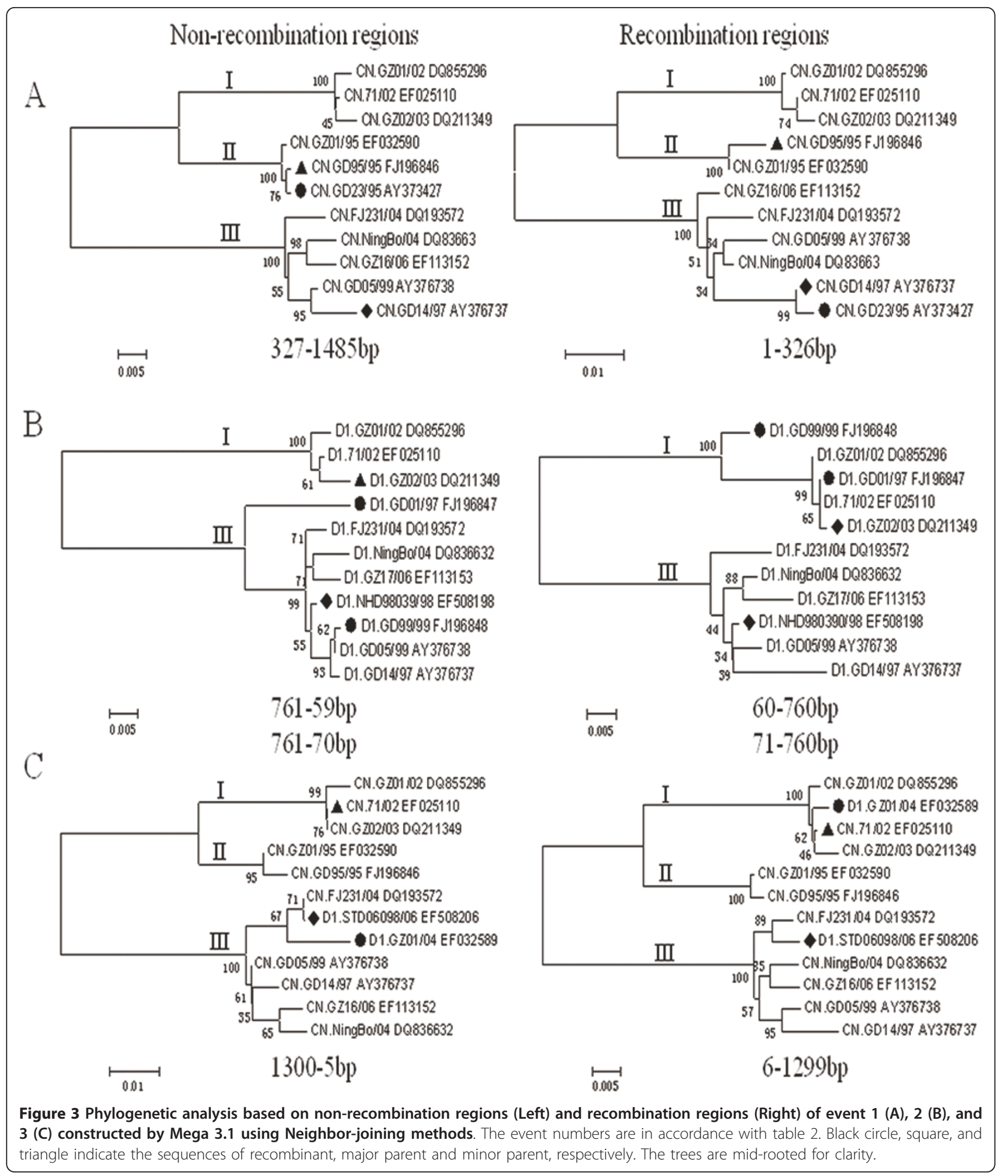

However, we found 22 intra-serotype potential recombination events (17 for DENV-1, 5 for DENV-2) (Table 3). Interestingly, for DENV-1, 13 of 17 potential recombination events happened in the strains isolated in 1995, 1997 and 1999 epidemics (Table 3), prior to which, in
1995, the severest dengue outbreak ever reported, indicating a possibility of viruses escaping human immunity by recombination following epidemic. However, whether the presumption makes sense or just a coincidence remains an open question. 
Table 3 Recombination events detected by RDP3 ${ }^{\dagger}$

\begin{tabular}{|c|c|c|c|c|c|}
\hline \multirow[t]{2}{*}{ Recombinant Event Number } & \multicolumn{2}{|c|}{$\begin{array}{l}\text { Breakpoint Positions in } \\
\text { Recombinant Sequence }\end{array}$} & \multirow[t]{2}{*}{ Recombinant Sequence(s) } & \multirow[t]{2}{*}{ Minor Parental Sequence } & \multirow[t]{2}{*}{ Major Parental Sequence } \\
\hline & Begin & End & & & \\
\hline 1 & 677 & $10104^{*}$ & D2.GD06/93 & D2.GD01/93 & D2.GD08/98 \\
\hline 2 & 5514 & $6548^{*}$ & D1.GD05/99 & D1.GD95/95 & D1.GD01/06 \\
\hline \multirow[t]{2}{*}{3} & $1^{*}$ & 7487 & D1.GD01/97 & D1.GD14/97 & D1.GD95/95 \\
\hline & & & D1.GD99/99 & D1.ZJ01/04 & D1.GZ01/95 \\
\hline \multirow[t]{2}{*}{4} & 900 & 1605 & D1.GD01/97 & D1.GZ71/02 & D1.GD14/97 \\
\hline & & & D1.GD99/99 & D1.GZ01/95 & D1.ZJ01/04 \\
\hline 5 & 5763 & 6401 & D1.GD99/99 & D1.GD66/03 & D1.GD01/06 \\
\hline 6 & $1^{*}$ & 466 & D1.GD05/99 & D1.GD23/95 & D1.GD99/99 \\
\hline 7 & 5585 & 6219 & D1.GD01/97 & D1.GD66/03 & D1.GD14/97 \\
\hline 8 & 749 & 1167 & D1.GD23/95 & D1.GD14/97 & D1.GD95/95 \\
\hline 9 & 8240 & 9343 & D2.GD08/98 & D2.GD01/93 & D2.GZ44/89 \\
\hline 10 & 5457 & $6201^{*}$ & D1.GZ71/02 & D1.GZ01/95 & D1.GD54/03 \\
\hline 11 & $1606^{*}$ & 2555 & D1.GD14/97 & D1.GD99/99 & D1.GD01/97 \\
\hline 12 & 3711 & 4913 & D2.GD01/01 & D2.GD06/93 & D2.ZS01/01 \\
\hline 13 & $2588^{*}$ & 2898 & D1.GD23/95 & D1.GD14/97 & D1.GZ01/95 \\
\hline 14 & 5171 & 5506 & D1.GD23/95 & D1.GD14/97 & D1.GD95/95 \\
\hline 15 & 7890 & 8795 & D1.GD01/97 & D1.GD99/99 & Unknown \\
\hline 16 & 2708 & 3121 & D1.GD01/06 & D1.GD54/03 & D1.ZJ01/04 \\
\hline 17 & 4889 & 5368 & D2.GD08/98 & D2.ZS01/01 & D2.GZ44/89 \\
\hline 18 & 9645 & $10146^{*}$ & D1.GD95/95 & D1.GD03/91 & D1.GZ01/95 \\
\hline 19 & $7890^{*}$ & 8385 & D1.GD99/99 & D1.GZ71/02 & Unknown \\
\hline 20 & 8820 & 9106 & D1.GD99/99 & D1.GD23/95 & D1.GD05/99 \\
\hline 21 & 2335 & $2398^{*}$ & D1.GD95/95 & Unknown & D1.GD23/95 \\
\hline 22 & $1043^{*}$ & $10104^{*}$ & D2.GD06/93 & D2.GD01/93 & D2.ZS01/01 \\
\hline
\end{tabular}

${ }^{\dagger}=22$ final recombination events resulting from at least two methods confirmation integrated in RDP3 package with a multiple comparison corrected P-value $<$ 0.01 and one-by-one manual checking. Only the closest major and minor parental sequences were shown in the table. Recombinants of DENV-1 were bolded for clarity.

* = The actual breakpoint position is undetermined (it was most likely overprinted by a subsequent recombination event).

Minor Parent $=$ Parent contributing the smaller fraction of sequence.

Major Parent $=$ Parent contributing the larger fraction of sequence.

Unknown = Only one parent and a recombinant need be in the alignment for a recombination event to be detectable.

\section{Conclusions}

Our study suggested that Dengue fever epidemic in Southern China over the past 30 years presents two important modes, 1) imported-cases-induced endemic prevalence; 2) endogenous epidemic outbreak with natural epidemic focuses. Recombination analysis showed that recombination plays an important role in dengue virus evolution and adaptation.

\section{Conflict of interests declaration}

The authors declare that they have no competing interests.

\section{Additional material}

Additional file 1: Table S1-S3. Overlapping PCR primers for DENV-1, DENV-2 and DENV-4 genome sequences

\section{Acknowledgements}

We thank Dr. Lin Wu for critical reading of the manuscript. This work was sponsored by Infectious Diseases Special Project, Minister of Health of China (2008ZX10004-001, 2009ZX10004-103).

\section{Author details}

${ }^{1}$ Key Laboratory of Genome Sciences and Information, Beijing Institute of Genomics, Chinese Academy of Sciences, Beijing, 100029, China.

${ }^{2}$ Guangzhou Medical Research Institute, Yan-ling, Guangzhou, 510507, China. ${ }^{3}$ Shenzhen Second People's Hospital, Shenzhen, 518035, China. ${ }^{4}$ College of Biotechnology, Southwest University, Chongqing, 400715, China. ${ }^{5}$ Beijing Genomics Institute in Shenzhen. Shenzhen, 518000, China.

\section{Authors' contributions}

WLW, HQZ, ZT and LCL carried out the genes sequencing and phlogenetics analysis. ZJB and MYF carried out the virus's culture and serotype identification. BHT and JHL participated in the samples detection. JWL and WJC designed the study, perform the data analysis and wrote the manuscript. All authors read and approved the final manuscript.

Received: 2 January 2011 Accepted: 26 June 2011

Published: 26 June 2011 


\section{References}

1. Gubler DJ: Dengue and dengue hemorrhagic fever. Clin Microbiol Rev 1998, 11:480-96.

2. Halstead SB: Dengue. Lancet 2007, 370:1644-52.

3. WHO: Dengue and dengue haemorrhagic fever. South-East Asia Region. Wkly Epidem Rec 1986, 61(27):205.

4. Drake JW, Holland JJ: Mutation rates among RNA viruses. Proc Natl Acad Sci USA 1999, 96:13910-3.

5. Thu HM, Lowry K, Jiang L, Hlaing T, Holmes EC, Aaskov J: Lineage extinction and replacement in dengue type 1 virus populations are due to stochastic events rather than to natural selection. Virology 2005, 336:163-72

6. Zhang C, Mammen MP Jr, Chinnawirotpisan P, Klungthong C, Rodpradit P, Monkongdee P, Nimmannitya S, Kalayanarooj S, Holmes EC: Clade replacements in dengue virus serotypes 1 and 3 are associated with changing serotype prevalence. J Virol 2005, 79:15123-30

7. Worobey M, Rambaut A, Holmes EC: Widespread intra-serotype recombination in natural populations of dengue virus. Proc Natl Acad Sci USA 1999, 96:7352-7.

8. Tolou HJG, Couissinier-Paris P, Durand JP, Mercier V, de Pina JJ, de Micco P, Billoir F, Charrel RN, de Lamballerie X: Evidence for recombination in natural populations of dengue virus type 1 based on the analysis of complete genome sequences. J Gen Virol 2001, 82:1283-90.

9. Uzcategui NY, Camacho D, Comach G, Cuello de Uzcategui R, Holmes EC, Gould EA: Molecular epidemiology of dengue type 2 virus in Venezuela: evidence for in situ virus evolution and recombination. J Gen Virol 2001, 82:2945-53.

10. Chen SP, Yu M, Jiang T, Deng YQ, Qin CF, Han JF: EQ: Identification of a recombinant dengue virus type 1 with 3 recombination regions in natural populations in Guangdong province, China. Arch Virol 2008, 153:1175-9.

11. Laille M, Deubel V, Sainte-Marie FF: Demonstration of concurrent dengue 1 and dengue 3 infection in six patients by the polymerase chain reaction. J Med Virol 1991, 34:51-4.

12. Li G, Yao JL, Peng WW, Wang F, Luo HR, Liao YH: Co-infection of dengue virus type 2 and 4 during 1993 epidemic in Guangdong province, China. Chin Med J 1995, 75:38-9.

13. Peng W, Man Y, Baochang F, Yongqiang D, Tao J, Hongyuan D: EQ: Simultaneous infection with dengue 2 and 3 viruses in a Chinese patient return from Sri Lanka. J Clin Virol 2005, 32:194-8.

14. Bharaj P, Chahar HS, Pandey A, Diddi K, Dar L, Guleria R, Kabra SK, Broor S: Concurrent infections by all four dengue virus serotypes during an outbreak of dengue in 2006 in Delhi, India. Virol J 2008, 5:1-5.

15. Xianghua Mao, Zaixing Zhang: Current situation of the dengue fever in China. Journal of Pathogen Biology 2007, 2:385-388.

16. The Collaborting Group for the Prevention and Treatment of Dengue Fever, Foshan, Guangdong: Etiologic and serologic investigation during an epidemic of dengue fever in Foshan District of Guangdong Province in 1978. Acta Microbiol Sinica 1981, 21:239-46.

17. Luo HM, He JF, Zheng K, Li LH, Jiang LM: Analysis on the epidemiologic features of Dengue fever in Guangdong province, 1990-2000. Chin J Epidemiol 2002, 23:427-430.

18. Liang WJ, He JF, Luo HM, Zhou HQ, Yang F, Zheng K: Epidemiologlcal analysis of dengue fever in Guangdong province, 2001-2006. South China Journal of Preventive Medicine 2007, 33:4-7.

19. Fan WF, Yu SR, Cosgriff TM: The reemergence of dengue in China. Rev Infect Dis 1989, 11:5847-53.

20. Fang M, Zhao W, Liu J: Molecular epidemiology of dengue fever. Chin J Epidemiol 2002, 23:148-50.

21. Ewing B, Hillier L, Wendl MC, Green P: Base-calling of automated sequencer traces using phred. I. Accuracy assessment. Genome Res 1998, 8:175-185.

22. Kumar S, Tamura K, Nei M: MEGA3: Integrated software for molecular evolutionary genetics analysis and sequence alignment. Brief Bioinform 2004, 5:150-163.

23. Martin DP, Posada D, Crandall KA, Williamson C: A modified bootscan algorithm for automated identification of recombinant sequences and recombination breakpoints. Aids Res Hum Retrov 2005, 21:98-102.

24. Martin DP, Williamson C, Posada D: RDP2: recombination detection and analysis from sequence alignments. Bioinformatics 2005, 21:260-2.
25. Padidam M, Sawyer S, Fauquet CM: Possible emergence of new Geminiviruses by frequent recombination. Virology 1999, 265:218-25

26. Posada D, Crandall KA: Evaluation of methods for detecting recombination from DNA sequences: Computer simulations. Proc Nat Acad Sci USA 2001, 98:13757-62.

27. Smith JM: Analyzing the mosaic structure of genes. J Mol Evol 1992 34:126-9.

28. Lindenbach $B D$, Rice $C M$ : Flaviviridae: the viruses and their replication. In Fields virology.. 4 edition. Edited by: Knipe DM, Howley PM. Lippincott Williams and Wilkins, Philadelphia, Pa; 2001:991-1041.

29. Mandl CW, Guirakhoo F, Holzman H, Heinz FX, Kunz C: Antigenic structure of the flavivirus envelope protein $\mathrm{E}$ at the molecular level, using tickborn encephalitis virus as a model. J Virol 1989, 63:564-571.

30. Lanciotti RS, Lewis JL, Gubler DJ, Trent DW: Molecular evolution and epidemiology of dengue-3 viruses. J Gen Virology 1994, 75:65-75.

31. Wittke V, Robb TE, Thu HM, Nisalak A, Nimmannitya S, Kalayanrooj S, Vaughn DW, Endy TP, Holmes EC, Aaskov JG: Extinction and rapid emergence of strains of dengue virus during an interepidemic period. Virology 2002, 301:148-156.

32. Aaskov J, Buzacott K, Field E, Lowry K, Berlioz-Arthaud A, Holmes EC: Multiple recombinant dengue type 1 viruses in an isolate from a dengue patient. J Gen Virol 2007, 88:3334-40.

doi:10.1186/1743-422X-8-322

Cite this article as: Wu et al:: Molecular epidemiology of dengue viruses in southern China from 1978 to 2006. Virology Journal 2011 8:322.

\section{Submit your next manuscript to BioMed Central and take full advantage of:}

- Convenient online submission

- Thorough peer review

- No space constraints or color figure charges

- Immediate publication on acceptance

- Inclusion in PubMed, CAS, Scopus and Google Scholar

- Research which is freely available for redistribution 